\title{
INTEROBSERVER VARIABILITY REGARDING KI67 PROLIFERATION INDEX EVALUATION IN GANGLIOGLIOMA AND ANAPLASTIC GANGLIOGLIOMA
}

\author{
Antonia C. LISIEVICI ${ }^{1}$, Florinel POP ${ }^{1,2}$, Simona BARBU ${ }^{1}$, Tiberiu A. GEORGESCU1,3凶, \\ Diana PASOV ${ }^{4}$, Mihai G. LISIEVICI ${ }^{4}$, Maria SAJIN ${ }^{1,5}$
}

${ }^{1}$ Department of Pathology, "Carol Davila" University of Medicine and Pharmacy, Bucharest, Romania

${ }^{2}$ Department of Pathology, "Carol Davila" Clinical Hospital, Bucharest, Romania

${ }^{3}$ Department of Pathology, National Institute for Mother and Child Health "Alessandrescu-Rusescu", Bucharest, Romania

${ }^{4}$ Department of Pathology, "Bagdasar-Arseni" Emergency Hospital, Bucharest, Romania

${ }^{5}$ Department of Pathology, Emergency University Hospital of Bucharest, Bucharest, Romania

Received 04 April 2021, Accepted 17 May 2021

https://doi.org/10.31688/ABMU.2021.56.2.03

\begin{abstract}
Introduction. Ganglioglioma represents a rare neuroglial tumour, affecting most frequently the pediatric population and arising in the temporal lobe. Based on the Ki67 proliferation index, gangliogliomas can be divided into benign and anaplastic.

The aim of our study was to assess the interobserver variability regarding the estimation of the Ki67 index, in order to establish if such a system could have a high reproducibility.
\end{abstract}

Material and methods. The proliferative activity of 25 cases including benign and anaplastic ganglioglioma has been revised through the Ki67 marker (clone SP6). The cases have been evaluated by five different pathologists with different degrees of experience, in order to test the reproducibility. Additionally, all the cases were evaluated for immunoreactivity for CD34 (clone QBEnd/10).

Results. Out of the 25 cases included in this study, $44 \%$ of them were diagnosed as anaplastic gangliogliomas.

\section{RÉSUMÉ}

Inter-observer variabilité concernant l'évaluation de l'indice de prolifération Ki67 dans le gangliogliome et le gangliogliome anaplasique

Introduction. Le gangliogliome représente une tumeur neurogliale rare, affectant le plus fréquemment la population pédiatrique et survenant dans le lobe temporal. Sur la base de l'indice de prolifération Ki67, les gangliogliomes peuvent être divisés en bénins et anaplasiques.

Le but de notre étude était d'évaluer la variabilité inter-observer concernant l'estimation de l'indice Ki67, afin d'établir si un tel système pouvait avoir une reproductibilité élevée.

Matériel et méthodes. Lactivité proliférative de 25 cas incluant les gangliogliomes bénin et anaplasique a été revue à travers le marqueur Ki67 (clone SP6). Les cas ont été évalués par cinq pathologistes différents avec différents degrés d'expérience, afin de tester la reproductibilité. De 
Best interobserver agreement was noted in cases with a low Ki67 index ( $<5 \%)$, namely in $85.71 \%$ of all benign gangliogliomas. Cases of anaplastic ganglioglioma which had a fatal outcome featured $\mathrm{Ki} 67$ values ranging from $2 \%$ to $45 \%$. In anaplastic ganglioglioma, the difference between the lowest and the highest assigned Ki67 value per case ranged from 4 to 25 , with a mean value of the differences of 11 .

Conclusions. Most cases of benign gangliogliomas can easily be identified through Ki67, with little interobserver variability. Similar results can be obtained for anaplastic gangliogliomas with high $\mathrm{Ki} 67$ values. Nonetheless, there is a small percentage of cases with relatively high Ki67 values and little reproducibility among pathologists.

Keywords: ganglioglioma, grading, anaplastic ganglioglioma.

\author{
List of abbreviations \\ WHO: World Health Organization \\ H\&E: Hematoxylin-eosin \\ SPSS: Statistical Package for the Social Sciences \\ IHC: Immunohistochemistry \\ DAB: 3,3'-Diaminobenzidine
}

\section{INTRODUCTION}

Ganglioglioma is a mixed neuroglial tumour arising most frequently in the temporal lobe of children and young adults ${ }^{1}$. The malignant counterpart of ganglioglioma, anaplastic ganglioglioma, can arise de novo, or by subsequent malignant transformation of the former ${ }^{2-3}$. Previous editions of the World Health Organization (WHO) Classification of Tumours of the Central Nervous System have proposed a three-tier system, which included also atypical ganglioglioma (WHO grade II). Nonetheless, the last two editions have excluded this entity, but do acknowledge that this category might exist, no clear cut histological criteria having been defined ${ }^{4}$.

Previously published studies have proposed different cut-off values of the Ki67 proliferation index, depending on which one can classify a tumour as benign or malignant. However, there is a lack of consistency regarding the cut-off value. Some authors consider a value of $5 \%$ as adequate for the diagnosis of atypical ganglioglioma, while others consider a value of $5-7 \%$ as consistent with the diagnosis of anaplastic ganglioglioma ${ }^{4-5}$.

The OBjective of THE Study was to analyse the interobserver variability encountered in the examination of the $\mathrm{Ki} 67$ proliferation index. This objective could allow future development of a new three tier plus, tous les cas ont été évalués pour la réponse immunologique pour CD34 (clone QBEnd / 10).

Résultats. Parmi les 25 cas inclus à cette étude, $44 \%$ d'entre eux ont été diagnostiqués comme gangliogliome anaplasique. La meilleure concordance inter-observer a été notée dans les cas à faible indice $\mathrm{Ki} 67$ ( $<5 \%$ ), à savoir dans $85,71 \%$ de tous les gangliogliomes bénins. Les cas de gangliogliome anaplasique ayant eu une issue fatale présentaient des valeurs de $\mathrm{Ki} 67$ allant de $2 \%$ à $45 \%$. Dans le gangliogliome anaplasique, la différence entre la valeur Ki67 attribuée la plus faible et la plus élevée par cas variait de 4 à 25 , avec une valeur moyenne des différences de 11.

Conclusions. La plupart des cas de gangliogliome bénin peuvent être facilement identifiés grâce à Ki67, avec une variabilité insignifiante inter-observer. Des résultats similaires peuvent être obtenus pour les gangliogliomes anaplasiques avec des valeurs Ki67 élevées. Néanmoins, il existe un petit pourcentage de cas aux valeurs de Ki67 relativement élevées et à reproductibilité diminuée estimés par les pathologistes.

Mots-clés: gangliogliome, gradation, gangliogliome anaplasique.

system for grading gangliogliomas, namely grade I, grade II and grade III.

\section{Material AND MEthods}

In this study we included 25 cases of ganglioglioma and anaplastic ganglioglioma, diagnosed during a period of 6 years (2015-2020), in the Pathology Department of the Emergency Clinical Hospital "Bagdasar-Arseni", Bucharest. The Hematoxilin-eosin (H\&E) stained slides have been reevaluated and new slides from the respective paraffin blocks were subsequently stained for Ki67 (clone SP6) and CD34 (clone QBEnd/10).

The Ki67 stained slides have been evaluated by 5 different pathologists, with different levels of experience: two senior pathologists, two specialists and one pathologist in training. Their experience in interpreting immunohistochemistry varied from 2 years to 20 years. None of the examining pathologists knew the initial diagnosis or the results given by the other colleagues, but the H\&E-stained slides of the cases were provided. Regarding CD34, the cases have been divided into four categories: cases which showed no reactivity for CD34, cases showing focal reactivity of single cells, cases with focal reactivity of clusters and cases with diffuse reactivity.

The clinical and imaging data have been acquired from the virtual and physical database of the hospital 


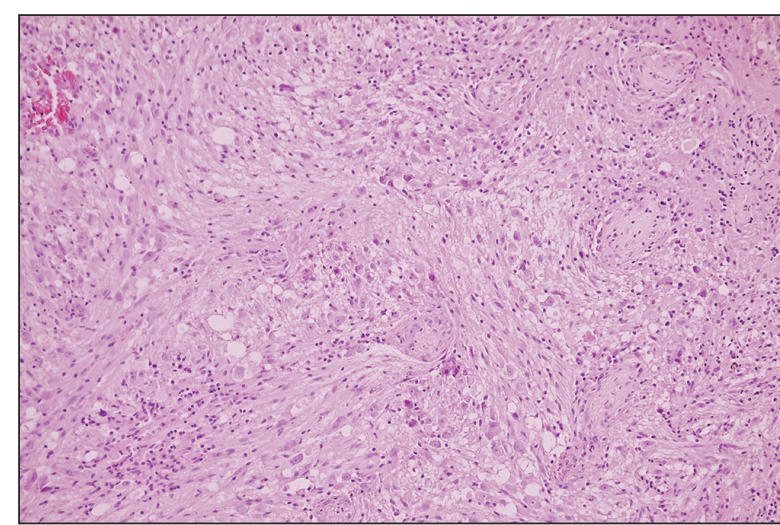

Fig. 1. Hematoxylin-eosin staining showing a benign ganglioglioma, with an abundant neuronal component, that includes scattered dysmorphic neurons (H.E., 200x).

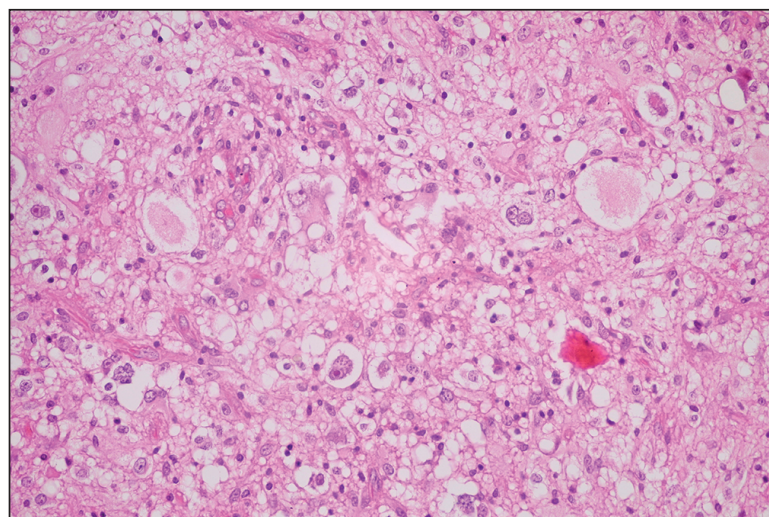

Fig. 3. Hematoxylin-eosin staining showing an anaplastic ganglioglioma with marked pleomorphism of both glial and neuronal component (H.E., 400x).

and follow up of the patients has been attained for a median of 48.6 months. The results provided by the examiners have been introduced in a Microsoft Excel sheet and further analysed through the SPSS software.

An approval was obtained from the local Ethics Committee of the Emergency Clinical Hospital "Bagdasar-Arseni", Bucharest, Romania (registration number 14110/12.04.2021).

\section{Results}

Out of the 25 cases included in this study, $44 \%$ were diagnosed as anaplastic gangliogliomas and 56\% as benign gangliogliomas, based solely on the $\mathrm{H} \& \mathrm{E}$ aspects (Fig. 1-3). The average age of the patients diagnosed with anaplastic ganglioglioma was 49.5 years, while those with benign ganglioglioma had an average of 37.7 years.

The Ki67 proliferation index showed variable results (Fig. 4-5). The best interobserver agreement has been observed in cases of benign ganglioglioma

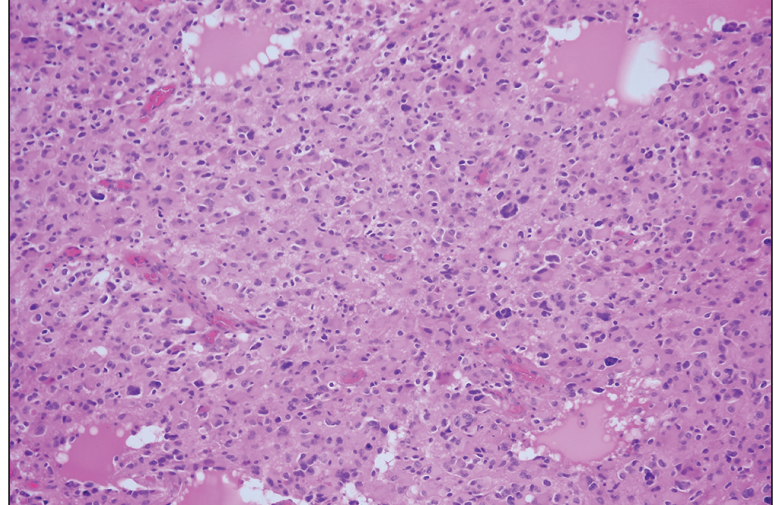

Fig. 2. Hematoxylin-eosin staining revealing an anaplastic ganglioglioma composed of dysmorphic neurons, interspersed within

a high-grade glial proliferation (H.E., 200x).

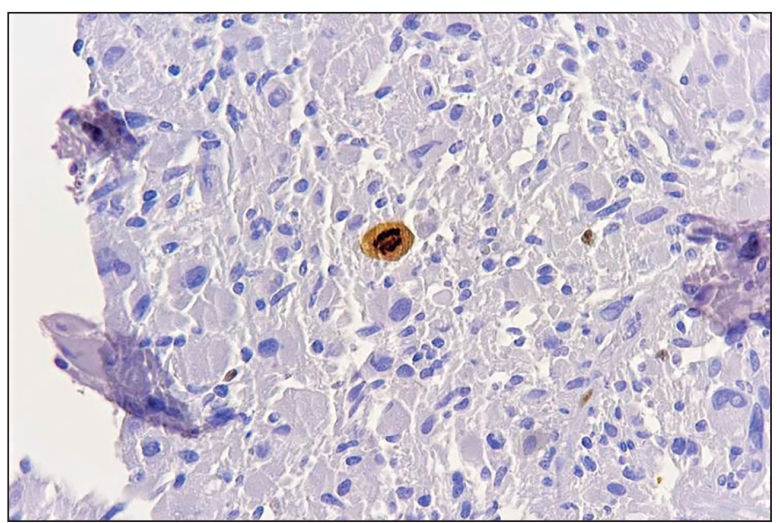

Fig. 4. Ki67 immunohistochemical stain of a benign ganglioglioma, showing a low proliferative index, but highlighting an atypical neuronal mitosis (IHC with DAB chromogen, 400x).

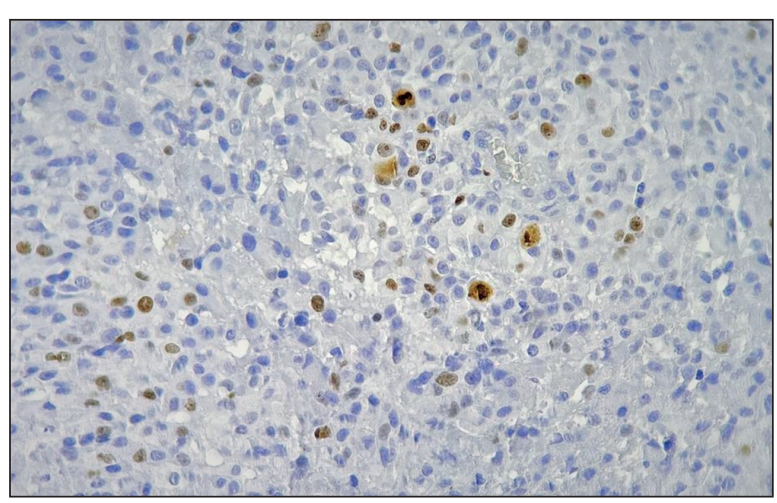

Fig. 5. Ki67 immunohistochemical stain of an anaplastic ganglioglioma that features a proliferative index of more than $20 \%$ (IHC with DAB chromogen, 400x).

with a Ki67 proliferation index of $1 \%$ or below, representing $42.86 \%$ of all benign gangliogliomas (Fig. 6). Even with a small degree of interobserver variability, values less than $5 \%$ were attained by all examiners to $85.71 \%$ of benign gangliogliomas. However, two cases (14.29\%) were assigned values varying from $10 \%$ 


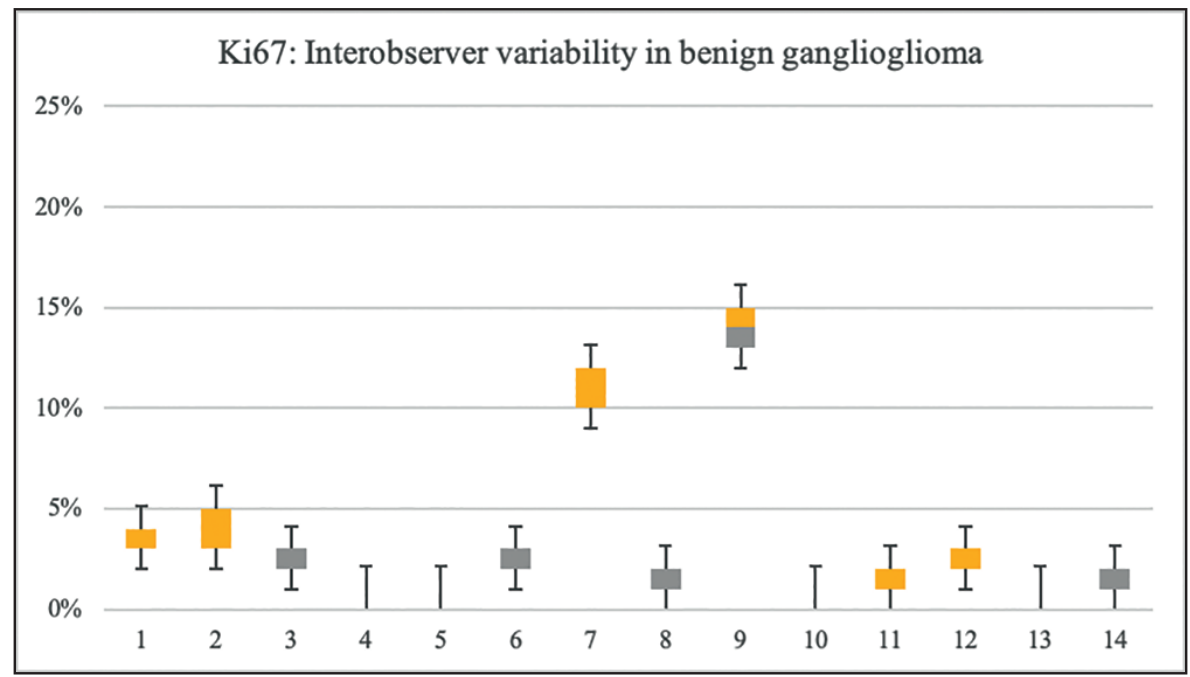

Fig. 6. Boxplot showing the interobserver variability in benign ganglioglioma

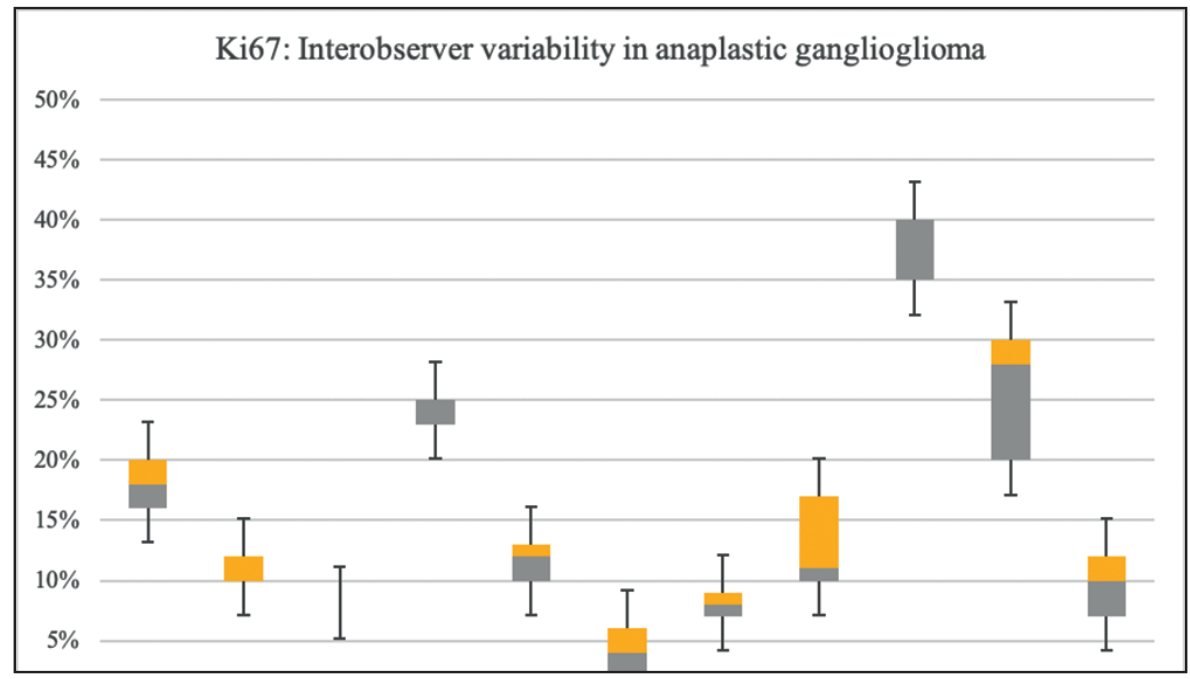

Fig. 7. Boxplot showing interobserver variability in anaplastic ganglioglioma.

Table 1. Comparison of the interobserver variability of Ki67 index interpretation between benign and malignant ganglioglioma.

\begin{tabular}{ccccc}
\hline \multirow{2}{*}{ Examinator } & \multicolumn{2}{c}{ Benign ganglioglioma } & \multicolumn{2}{c}{ Anaplastic ganglioglioma } \\
\cline { 2 - 5 } & $\begin{array}{c}\text { Interval of Ki67 index } \\
\text { values }\end{array}$ & $\begin{array}{c}\text { Average of Ki67 index } \\
\text { values per examinator }\end{array}$ & $\begin{array}{c}\text { Interval of Ki67 index } \\
\text { values }\end{array}$ & $\begin{array}{c}\text { Average of Ki67 index values per } \\
\text { examinator }\end{array}$ \\
\hline Examinator 1 & $1-13 \%$ & $4 \%$ & $7-45 \%$ & $18 \%$ \\
\hline Examinator 2 & $1-15 \%$ & $4 \%$ & $6-40 \%$ & $20 \%$ \\
\hline Examinator 3 & $1-14 \%$ & $3 \%$ & $2-35 \%$ & $14 \%$ \\
\hline Examinator 4 & $1-12 \%$ & $3 \%$ & $3-28 \%$ & $12 \%$ \\
\hline Examinator 5 & $1-20 \%$ & $4 \%$ & $2-40 \%$ & $14 \%$ \\
\hline
\end{tabular}

to $15 \%$, respectively from $12 \%$ up to $20 \%$. Upon follow-up, the latter case did indeed recur 5 months later as an anaplastic ganglioglioma, thus justifying the high proliferation rate from the initial excision, which was in opposition with the bland histological aspects.
The low proliferation index evaluated in benign gangliogliomas, strongly correlated with the high survival rate of $91.67 \%$. The only case that had a fatal outcome, from those with a low Ki67 index, was in a patient that encountered perioperative complications and died in the first days after the surgery. The 


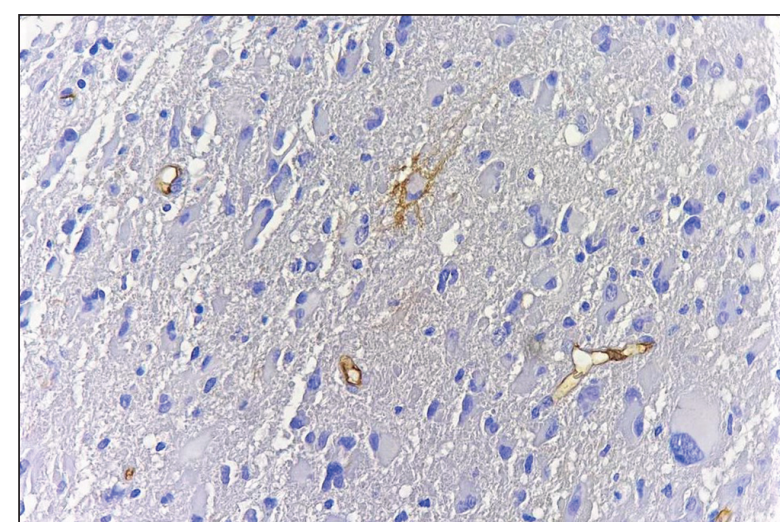

Fig. 8. CD34 immunohistochemical stain revealing solitary neurons that present membranous immunoreactivity for this marker with discrete spreading into the surrounding tissue. (IHC with DAB chromogen, 400x)

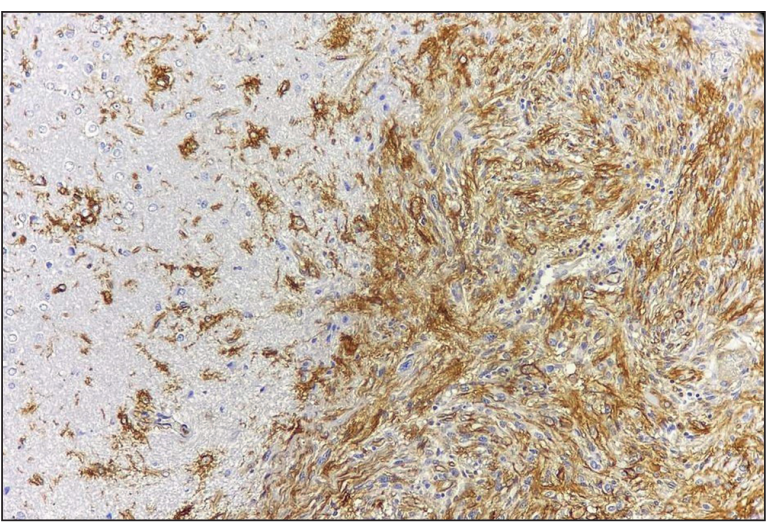

Fig. 10. CD34 immunohistochemical stain showing diffuse immunoreactivity of the tumoral area, but also revealing scattered immunoreactive neurons that are spreading into the surrounding tissue (IHC with DAB chromogen, 200x).

overall survival rate of patients diagnosed on histology with benign ganglioglioma was of $85.71 \%$.

The proliferation index was also evaluated in eleven cases of anaplastic ganglioglioma, and the results varied from case to case, ranging from very low values (2-7\%), to very high ones (20-45\%) (Fig. 7). In opposition with the cases of benign ganglioglioma, high values of Ki67 did not correlate with survival. Upon a separate analysis of patients that have a high survival rate upon follow-up, the Ki67 proliferation index showed a high proliferative rate, varying from $5 \%$ to $35 \%$. On the other hand, cases that had a fatal outcome, had Ki67 proliferation indices spread upon a wider range of values, from $2 \%$ to $45 \%$. This result proves that not even low Ki67 proliferation indices are specific for benign gangliogliomas (Table 1). The overall survival rate for anaplastic ganglioglioma was of $36.36 \%$. When evaluating the mean value of all the minimal Ki67 attributed in all cases of anaplastic

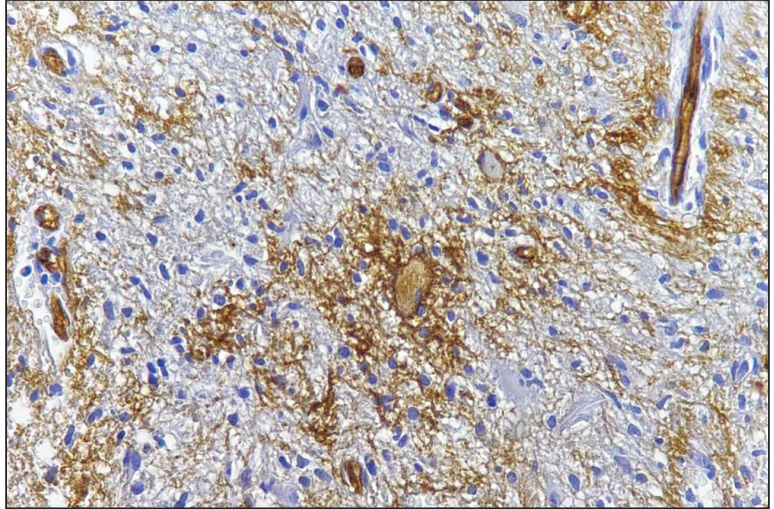

Fig. 9. CD34 immunohistochemical stain showing immunoreactivity of groups of neurons, without the constitution of diffuse areas (IHC with DAB chromogen, 400x).

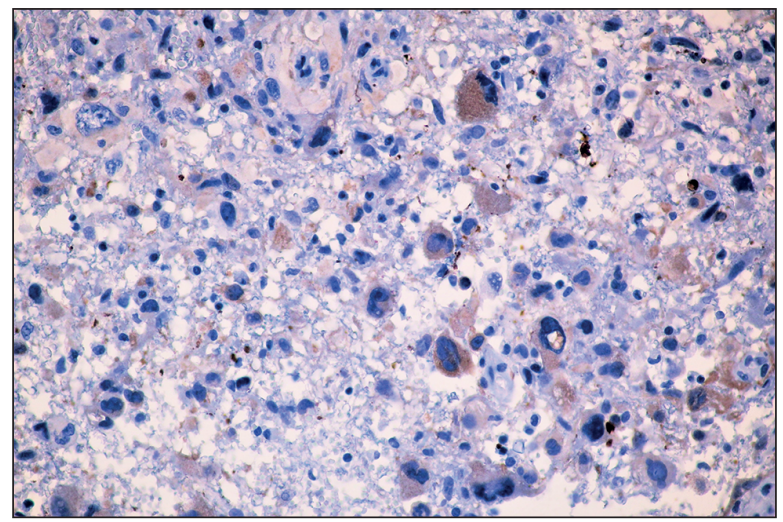

Fig. 11. Chromogranin immunohistochemical staining of an anaplastic ganglioglioma, showing immunoreactivity of dysmorphic neurons (IHC with DAB chromogen, 400x).

ganglioglioma, an average of $10 \%$ is observed. When applying the same algorithm for the highest values of Ki67 indices for all cases of anaplastic ganglioglioma, an average of 21 is observed.

Regarding the interobserver variability in evaluation of the Ki67 proliferation index, in benign ganglioglioma, the biggest difference per case, between the lowest and the highest assigned values was 8 and the mean difference was 2. In anaplastic ganglioglioma, the difference between the lowest and the highest assigned Ki67 value per case, ranged from 4 to 25 , with a mean difference of 11 .

CD34 expression has been evaluated and interpreted according to the algorithm proposed by Blumcke et $\mathrm{al}^{6}$, in which three patterns of staining can be observed: solitary cells (Fig. 8), clusters of cells (Fig. 9) and diffuse staining (Fig. 10). Overall CD34 expression was noticed in $88 \%$ of cases, with most cases expressing diffuse immunoreactivity for 
CD34 (40.91\%), closely followed by the cluster pattern $(31.82 \%)$ and solitary cells reactivity (27.27\%).

On a separate analysis of anaplastic ganglioglioma, we observe that an equal percentage of cases showed diffuse staining $(36.36 \%)$ and cluster pattern of staining (36.36), with only $9.09 \%$ of cases showing immunoreactivity in solitary cells. Out of all anaplastic gangliogliomas, $18.18 \%$ of cases showed no immunoreactivity for CD34. The presence of neuronal cells was also highlighted with Chromogranin immunohistochemical staining (Fig. 11).

Cases of benign ganglioglioma showed more diverse results regarding CD34 pattern of staining. Solitary cell immunoreactivity $(35.71 \%)$ and diffuse pattern of staining $(35.71 \%)$ were most frequently encountered, followed by cluster pattern of staining $(21.43 \%)$ and cases that showed no immunoreactivity $7.14 \%$.

\section{Discussion}

Ganglioglioma is a rare tumor, affecting most commonly the pediatric population. It is frequently associated with the presence of epileptic seizures and has a predilection for the temporal lobe. Histologically, it is characterized by the presence of a mixed neuronal and glial cellular proliferation and frequently associates Rosenthal fibres and eosinophilic bodies? ${ }^{7}$. The glial component of the tumour is usually represented by a pilocytic astrocytoma ${ }^{8}$, but cases featuring a fibrillary, gemistocytic or protoplasmatic astrocytoma component have been described $^{9-11}$. The neuronal component is composed out of large cells with eosinophilic, abundant cytoplasm and large nuclei, that can be either scattered or may represent the majority of the tumour. The neurons are usually dysmorphic, can be binucleated and may associate ganglioig precursors ${ }^{12}$. However, the benign behaviour of this tumour has been largely discussed in the scientific literature, especially due to their malignant transformation potential. This situation, although rare, has frequently been reported in the literature and was also been encountered in the present study ${ }^{1,3,13}$.

Due to their malignant transformation potential, the diagnosis of atypical ganglioglioma has been proposed and different authors have proposed either a histological approach, an immunohistochemical or an imaging approach to this diagnosis ${ }^{14-15}$. Regarding the establishment of a possible threshold for Ki67 proliferation index, most authors agree that a value below $3 \%$ is consistent with the diagnosis of benign ganglioglioma and that a value below $2 \%$ correlates greatly with the survival ${ }^{16-17}$. Our study endorses this estimation, with $87.51 \%$ of benign ganglioglioma showing a low proliferative index, below $5 \%$, independent of the examining pathologist, or of their degree of experience. Also, authors have observed that these cases only show immunoreactivity of the glial component and not the neuronal on $\mathrm{e}^{18}$. On the other hand, most authors consider that a Ki67 proliferation index above $10 \%$ is suggestive for the anaplastic character of the proliferation ${ }^{17,19,20}$. In our study, only $63.64 \%$ of cases featured a Ki67 proliferation rate above $10 \%$, as estimated by 4 out of 5 pathologists (interobserver agreement). Additionally, 2 cases (14.29\%) of benign ganglioglioma also showed a Ki67 proliferation rate above $10 \%$. Nonetheless, one of those two cases did indeed progress to malignant transformation.

Although the current WHO classification of the central nervous system does not indicate a specific Ki67 proliferation index value for atypical ganglioglioma, authors have proposed a threshold of $5 \%$, above which gangliogliomas can be assigned the designation of atypical ${ }^{5}$. Hirose et al also noticed that a high proliferation index does indeed corelate with the presence of anaplasia and with a high degree of recurrences ${ }^{12}$. Our analysis confirms this hypothesis, considering that cases of benign ganglioglioma with bland histopathological features but a high Ki67 rate, subsequently recurred as an anaplastic ganglioglioma. Another notable observation was made by Prayson et al, who noticed that the recurrences typically have a higher proliferative activity when compared to the primary tumour ${ }^{21}$. This affirmation was also confirmed in the cases included in our study, which featured a Ki67 proliferative index higher with 4 up to 8 percent in the recurrences.

Blumcke et al observed that there exists a particular pattern of staining encountered in ganglioglioma. Cellular neoplastic elements show surface membrane immunoreactivity, which can be divided into three types of staining: diffuse homogeneous, clusters of cells and isolated tumoral cells called ,satellites" ${ }^{5-6}$. The same author has observed that this does not usually apply to anaplastic ganglioglioma, which usually express CD34 in a minor proportion ${ }^{5}$. However, other scientific articles do not endorse this theory. Zanello et al observed that $94 \%$ of all anaplastic gangliogliomas express CD34 in the form of solitary isolated cells and $16 \%$ feature a diffuse pattern of staining ${ }^{22-23}$. Our study also noticed a relatively high overall immunoreactivity for CD34 in anaplastic ganglioglioma (81.82\%), the large majority of cases showing either the diffuse $(36.36 \%)$ or the cluster pattern of staining $(36.36 \%)$.

Published scientific articles have observed that the cluster pattern of staining can be encountered in $55.5 \%$, while others noticed it in up to $74 \%$ of 
cases $^{6,24}$. The diffuse pattern of staining can be encountered in up to $55 \%$ of cases $^{25}$. Including both the cases of anaplastic and benign gangliogliomas, in our study the cluster pattern of staining was present in $28 \%$ of cases, while the diffuse pattern was encountered in $36 \%$ of all cases.

\section{Conclusions}

In conclusion, the best interobserver agreement can be attained for cases of benign gangliogliomas, with a Ki67 proliferation index below 5\%. A low proliferative index also correlates with a high survival rate of those patients. Cases with high Ki67 index also had an adequate interobserver agreement and also correlated with the mortality rate. Nonetheless, our study shows that there are cases of apparently benign gangliogliomas with a high proliferative rate, that may progress to anaplastic transformation, and also cases of anaplastic ganglioglioma with a proliferative rate below $10 \%$, that may have a fatal outcome. The reintroduction of grade II (atypical) ganglioglioma could be a solution to this problem, and in order to assign this grade, one should take into account both histological aspects, as well as the proliferative index. Further studies, on larger number of cases should be conducted, in order to verify the reproducibility of this hypothesis. Additionally, the CD34 expression can be observed in both benign and anaplastic ganglioglioma and did not correlate with the survival rate or disease-free survival.

\section{Author contributions}

Conceptualization, A.L., M.L., M.S.; methodology, A.L., T.G., validation, M.S., formal analysis, F.P., S.B., T.G., D.P., M.L.; resources, A.L., F.P. All the authors have read and agreed with the final version of the article.

\section{Compliance with Ethics Requirements}

"The authors declare no conflict of interest regarding this article"

"The authors declare that all the procedures and experiments of this study respect the ethical standards in the Helsinki Declaration of 1975, as revised in 2008(5), as well as the national law. Informed consent was obtained from all the patients included in the study. The study was conducted in accordance with the principles of good clinical practices (GCP) and reviewed and approved by the institutional review board (IRB) 00006384 ."

"No funding for this study"

\section{Acknowledgements}

None

\section{References}

1. Rosselló A, Plans G, Vidal-Sarró N, Fernández-Coello A, Gabarrós A. Ganglioglioma progression to combined anaplastic ganglioglioma and anaplastic pleomorphic xanthoastrocytoma. Case report and literature review. World Neurosurgery 2017;108: 996.e17-996.e25.

2. Lee CC, Wang WH, Lin CF, et al. Malignant transformation of supratentorial ganglioglioma. Clinical Neurology and Neurosurgery 2012;114(10):1338-42.

3. Riesberg G, Bathla G, Gupta S, Watal P, Moritani T. Malignant transformation and leptomeningeal spread of recurrent ganglioglioma: case report and review of literature. Clinical Imaging 2018; 48:7-11.

4. Becker AJ, Wiestler OD, Figarella-Branger D, Blümcke I, Capper D. Neuronal and mixed neuronal-glial tumours. In WHO Classification of Tumours of the Central Nervous System, 4th ed, Louis DN, Ohgaki H, Wiestler OD, Cavenee WK (eds). Lyon, International Agency for Research on Cancer, 2016:138-141.

5. Blümcke I, Wiestler OD. Gangliogliomas: An intriguing tumor entity associated with focal epilepsies. Journal of Neuropathology $\mathcal{E}$ Experimental Neurology 2002;61(7):575-84.

6. Blümcke I, Giencke K, Wardelmann E, et al. The CD34 epitope is expressed in neoplastic and malformative lesions associated with chronic, focal epilepsies. Acta Neuropathologica 1999;97(5):481-490.

7. Lisievici AC, Georgescu TA, Pasov D, Tascu A, Lisievici MG, Sajin M. Grading gangliogliomas: a short case series with clinico-imagistic and immunohistopathological correlations. Maedica (Bucharest) 2018;13(3):241-249.

8. Tuan TA, Duc NM. Giant cerebellar ganglioglioma mimicking a pilocytic astrocytoma. Journal of Clinical Imaging Science 2021;11:3.

9. Courville CB. Ganglioglioma: Tumor of the central nervous system; review of the literature and report of two cases. Archives Neurology E Psychiatry 1930;24(3):439-491.

10. Kalyan-Raman UP, Olivero WC. Ganglioglioma: A correlative clinicopathological and radiological study of ten surgically treated cases with follow-up. Neurosurgery 1987; 20(3): 428-433.

11. Nair V, Suri VS, Tatke M, Saran RK, Malhotra V, Singh D. Gangliogliomas: A report of five cases. Indian Journal of Cancer 2004;41(1):41-6.

12. Hirose T, Scheithauer BW, Lopes MB, Gerber HA, Altermatt HJ, VandenBerg SR. Ganglioglioma: an ultrastructural and immunohistochemical study. Cancer 1997; 79(5):989-1003.

13. Lummus SC, Aisner DL, Sams SB, Foreman NK, Lillehei $\mathrm{KO}$, Kleinschmidt-DeMasters BK. Massive dissemination from spinal cord gangliogliomas negative for BRAF V600E: Report of two rare adult cases. American Journal of Clinical Pathology 2014;142(2): 254-260.

14. Dorne HL, O'Gorman AM, Melanson D. Computed tomography of intracranial gangliogliomas. AJNR American Journal of Neuroradiology 1986;7(2):281-5.

15. Dapaah A, Biswas S, Srikandarajah N, Crooks D, Das K, Farah JO. Serial imaging and management of ganglioglioma with unusual presentation and meningeal spread. Acta Neurochirurgica (Wien) 2017; 159(3): 481-483.

16. Di Rocco C, Tamburrini G. Ganglioglioma, in Neuro-Oncology of CNS Tumors, Tonn JC, Grossman SA, Rutka JT, Westphall M (eds). Berlin, Springer, 2006: 393-400.

17. Zieman G, Dardis C, Gomes A, Scheck A, Eschbacher J, Ashby L. Predicting progressive gangliogliomas: early 
identification of those at risk in a sample of 156 consecutive patients treated at the Barrow Neurological Institute (S22.004). Neurology 2014; 82(10).

18. Rodríguez FJ. Neuronal and glioneuronal neoplasms. In Diagnostic pathology. Neuropathology, 2nd ed, Kleinschmidt-DeMasters B, Rodríguez FJ, Tihan T (eds). Elsevier, 2016:168-177.

19. Hayat MA. Tumors of the Central Nervous System. In Lymphoma, Supratentorial Tumors, Glioneuronal Tumors, Gangliogliomas, Neuroblastoma in Adults, Astrocytomas, Ependymomas, Hemangiomas, and Craniopharyngiomas. volume 9, Springer, 2012:137-164.

20. Brat DJ. Neuronal and mixed neuronal-glial tumours. In Greenfield's neuropathology, 9th ed, Love S, Budka H, Ironside JW, Perry A (eds). CRC Press, 2015:1727-1731.

21. Prayson RA, Khajavi K, Comair YG. Cortical architectural abnormalities and MIB1 immunoreactivity in gangliogliomas: A study of 60 patients with intracranial tumors. Journal of Neuropathology $\mathcal{E}$ Experimental Neurology 1995;54(4):513520.

22. Zanello M, Pages M, Tauzie'de-Espariat A, et al. Clinical, imaging, histopathological and molecular characterization of anaplastic ganglioglioma. Journal of Neuropathology $\mathcal{E}$ Experimental Neurology 2016;75(10):971-980.

23. Blümcke I. Brain tumors associated with early epilepsy onset. In Surgical neuropathology of focal epilepsies: textbook and atlas, Blümcke I, Sarnat HB, Coras R (eds). John Libbey Eurotext 2015:54-66.

24. Chappé C, Padovani L, Scavarda D, et al. Dysembryoplastic neuroepithelial tumors share with pleomorphic xanthoastrocytomas and gangliogliomas BRAF(V600E) mutation and expression. Brain Pathology 2013;23(5):574-583.

25. Deb P, Sharma MC, Tripathi M, Sarat Chandra P, Gupta A, Sarkar C. Expression of CD34 as a novel marker for glioneuronal lesions associated with chronic intractable epilepsy. Neuropathology and Applied Neurobiology 2006;32(5):461-468. 